[0212-7199 (2005) 22: 1; pp 15-20] ANALES DE MEDICINA INTERNA Copyright (C) 2005 ARAN EDICIONES, S.L.

AN. MED. INTERNA (Madrid) Vol. $22, \mathrm{~N}^{\circ} 1, \mathrm{pp} .15-20,2005$

\title{
Impacto de la ola de calor de 2003 en el Hospital de Riveira (A Coruña)
}

\author{
I. VILLAMIL CAJOTO, J. A. DÍAZ PEROMINGO, G. VILLACIAN VICEDO', \\ J. SÁNCHEZ LEIRA, F. GARCÍA SUÁREZ, J. SABORIDO FROJÁN, M. IGLESIAS \\ GALLEGO
}

Fundación Pública Hospital de Barbanza. Riveira, A Coruña. ${ }^{I}$ Estadístico. Universidad Complutense. Madrid

HEALTH IMPACT OF 2003 HEAT WAVE AT HOSPITAL DE RIVEIRA (A CORUÑA)

\section{RESUMEN}

Objetivo: El pasado verano de 2003 se vivió una situación atmosférica considerada como ola de calor. Es conocida la importancia de los factores ambientales sobre enfermos portadores de patologías crónicas, aunque existen pocos trabajos que analicen los derivados del exceso de calor, como los que estudiamos en este documento.

Método: Evaluamos el impacto de las altas temperaturas en los ingresos y la mortalidad en el área de Riveira, A Coruña, durante el periodo entre el 15 de julio y el 15 de agosto de 2003.

Resultados: La temperatura media máxima en este periodo se fue de $26,1^{\circ} \mathrm{C}$. Durante este periodo ingresaron 137 pacientes de edades comprendidas entre los 16 y los 93 años. Fallecieron 16 pacientes. Se identificaron 54 casos de ingresos relacionados con efecto del calor $(38,7 \%)$, con 8 fallecimientos en este grupo. Ningún caso pudo atribuirse a golpe de calor. Los principales factores de riesgo identificados en los ingresados por efecto del calor, fueron patología pulmonar previa y obesidad.

Conclusión: Si bien los efectos del calor son difíciles de evaluar, su importancia es clara. Es necesario adoptar medidas para identificar la población de riesgo y reducir los efectos por este fenómeno atmosférico dado que los pacientes de riesgo son altamente prevalentes en nuestra práctica diaria.

PALABRAS CLAVE: Golpe de calor.Ola de calor. Temperatura. Admisión hospitalaria.

\begin{abstract}
Objectives: Last summer 2003 took place an atmospheric situation considered as a heat wave. Importance of environmental factors in chronic diseases is well known, but few works analyse those of heat excess as we do in this paper.

Methods: We studied the effects of high temperatures on admission and mortality in Riveira, A Coruña, Spain, during the period from July 15th to august 15th 2003.

Results: Mean maximum temperature in this period was $26.1{ }^{\circ} \mathrm{C}$. One hundred and thirty seven patients were admitted, age ranges between 16 and 93 years old. Sixteen died. Fifty four admissions (38, $7 \%)$ were identified as related with heat wave and of those patients, 8 died. No heat stroke cases were identified. Main risk factors in our heat related admitted population, were previous lung disease and overweight.

Conclusion: Heat related pathology is difficult to evaluate although it is quite important. It seems to be necessary to take measures in order to identify population at risk and reduce the effects related to this environmental factor since high risk patients are quite common in our daily practice.
\end{abstract}

KEY WORDS: Heat illness. Heat wave. Temperature. Hospital admission.

Villamil Cajoto I, Díaz Peromingo JA, Villacian Vicedo G, Sánchez Leira J, García Suárez, F, Saborido Froján J, Iglesias Gallego M. Impacto de la ola de calor de 2003 en el Hospital de Riveira (A Coruña). An Med Interna (Madrid) 2005; 22: 15-20.

\section{INTRODUCCIÓN}

El organismo humano presenta un equilibrio fisiológico que puede alterarse por una serie de agentes externos patógenos. Los más importantes son los biológicos (virus, bacterias, etc.), los químicos (orgánicos o inorgánicos) y los físicos (definidos en función del entorno ecológico en el que se encuentra el ser vivo, entre os que destacan: las variaciones de iluminación, ionización, presión atmosférica, presiones parciales de oxígeno y agua y la temperatura) (1). Los cambios de magnitud y frecuencia de los fenómenos meteorológicos, ocasionan grandes impactos sobre la salud en las poblaciones desencadenando catástrofes o emergencias. Las más frecuentes en nuestra latitud son las olas de calor, las inundaciones y las sequías. La capacidad de adaptación a los cambios ambientales ha permitido al hombre la búsqueda de nuevos entornos habitables. Sin embargo esta habilidad de adaptación está limitada y en condiciones extremas la supervivencia se encuentra comprometida.

En estas condiciones extremas, en el caso de exceso de calor, pueden presentarse, los llamados golpes de calor que se caracterizan por una situación grave de fracaso multiorgánico,

Trabajo aceptado: 1 de octubre de 2004

Correspondencia: Iago Villamil Cajoto. Servicio de Medicina Interna. Hospital Clínico Universitario. R. Choupana, s/n. 15706 Santiago de Compostela (A Coruña).e-mail: iago.villamil.cajoto@sergas.es 
han sido bien definidos y confirmada sobradamente su relación con incrementos en la morbimortalidad (2-4). Estos casos parecen estar infraestimados dada la dificultad para discriminar el papel del exceso de calor en alguna de sus manifestaciones clínicas, particularmente trombosis coronaria o cerebral una vez que el cuerpo no se presenta con temperaturas extremas en ambientes calurosos (5). Existen una serie de factores que parecen favorecer el golpe de calor clásico $(4,6)$ (Tabla I).

\section{TABLA I}

\section{FACTORES DE RIESGO PARA LESIONES POR CALOR $(4,6)$}

\section{Deshidratación}

\section{Obesidad}

Ropa pesada o impermeable

Condición física deficiente

Enfermedad cardiovascular

Lesiones cutáneas (esclerodermia, quemaduras, eczema, psoriasis, trastornos de glándulas sudoríparas)

\section{Edades extremas}

Falta de movilidad

Enfermedad febril

Hipertiroidismo

Alcoholismo

Drogas (cocaína, anfetaminas, opiáceos, LSD)

Condiciones socioeconómicas (falta de aire acondicionado, vivir en los pisos superiores de edificios altos)

Esfuerzo prolongado en calor (trabajadores altos hornos, mineros, bomberos, atletas, reclutas militares, trabajadores de rescate y salvamento)

Medicamentos (antipsicóticos, anticolinérgicos, bloqueadores de los canales del calcio, bloqueantes beta, diuréticos, agonistas alfa, simpáticomiméticos)

Por otro lado hay evidencia bien conocida de variación en la mortalidad en relación estacional, de hecho algunos de estos cambios periódicos son fundamentales en la cadencia estacional de ciertas patologías. Las patologías respiratorias y cardiocirculatorias son las más relacionadas con la estacionalidad (7). Pero no solo el descenso térmico ha demostrado ser un factor determinante como podría parecer, si no que los cambios anormalmente altos en las temperaturas también se relacionan con aumento de la mortalidad. El fracaso en la termorregulación y la alteración en la modulación de la respuesta inflamatoria y de estrés facilitan la progresión del exceso de calor al golpe de calor por mecanismos poco conocidos de respuesta celular y molecular que probablemente merezcan un estudio más profundo (3).

Existen evidencias cada vez mayores del llamado cambio climático. Este supone en definitiva un aumento global de las temperaturas en la superficie terrestre como consecuencia de los diversos factores que incrementan la producción de los niveles de dióxido de carbono, por lo que esta situación de incremento en la temperatura ambiente puede a largo plazo tener importancia sobre ciertas patologías (8).

La olas de calor (definidas como periodos superiores a tres o más días con temperaturas del aire de al menos $32,2{ }^{\circ} \mathrm{C}$
-90 ${ }^{\circ} \mathrm{F}-$-) (9), son fenómenos meteorológicos que contribuyen de forma significativa en incrementos de mortalidad. Recientemente esta situación atmosférica vivida en 2003, ha sido considerada la estación mas calurosa en los últimos 90 años, siendo estos últimos años (1901-2000) los mas calurosos desde el año 1500 (10). Sobre la capacidad de adaptación a los cambios de temperatura, un estudio multicéntrico en regiones templadas y frías europeas, concluye que la mortalidad relacionada con el calor se produce a mayores temperaturas en regiones más calurosas que en las mas templadas o frías, sin que esto signifique un aumento en la mortalidad global en áreas calurosas (11). Diversos estudios epidemiológicos describen la relación entre la temperatura ambiental y la mortalidad, y así se conoce que los periodos de tiempo prolongados con temperaturas altas se asocian a mayores impactos en la mortalidad de lo que cabría esperarse de una relación lineal, probablemente por la persistencia del estrés térmico que se extiende a las temperaturas nocturnas (12). En Holanda, se demuestran cifras de mortalidad menores en días en los que la temperatura máxima se encuentra en torno a los $20-25{ }^{\circ} \mathrm{C}(13)$. Se considera que en el Reino Unido la mortalidad es menor cuando la temperatura media diaria está en torno a los $17-18^{\circ} \mathrm{C}$ (14). En nuestro país existe también evidencia documentada de asociación entre temperatura y mortalidad, determinándose la temperatura en la cual la mortalidad alcanza un mínimo en los, $24^{\circ} \mathrm{C}$ en un estudio realizado en Valencia (15).

La ola de calor del pasado verano de 2003 ha tenido importante difusión mediática y en algunos países existen datos publicados concretos, pero hasta la fecha no existen datos publicados en España sobre las repercusiones sanitarias directas, salvo comunicados de prensa, por lo que presentamos este estudio sobre el impacto en nuestra área sanitaria con datos para la discusión.

\section{PACIENTES Y MÉTODOS}

Se realizó la revisión sistemática de todos los ingresos en el Servicio de Medicina Interna del Hospital da Barbanza, Riveira, A Coruña. Se revisaron así mismo los exitus en el mismo periodo de estudio en los años anteriores. El Servicio de Medicina Interna cuenta con 41 camas. El área sanitaria que cubre, abarca 4 ayuntamientos próximos atendiendo a unos 65.000 usuarios. El área sanitaria de nuestro centro es fundamentalmente una zona costera con temperaturas medias en el periodo de verano de $22{ }^{\circ} \mathrm{C}$, con una humedad relativa ambiental del $87 \%$ y presión atmosférica de $1014 \mathrm{hPa}$. Se trata de la única unidad de hospitalización del área médica del centro. El estudio se realizó durante el periodo entre el 15 de julio de 2003 y el 15 de agosto de 2003 coincidiendo con el pico máximo de la ola de calor de ese verano en Galicia. Se realizó una valoración de los índices de hospitalización en relación con las curvas de temperaturas según datos oficiales de Meteogalicia en el observatorio de A Curota (Riveira) situado a unos $3 \mathrm{~km}$ del centro hospitalario. Se compararon las cifras de ingresos y la morbimortalidad con los mismos periodos de años anteriores.

Se consideraron ingresos en relación a la ola de calor, a aquellos pacientes con patologías de base que por sus características está sobradamente reconocido el papel del calor ambiental como factor precipitante, en las que no existían desencadenantes definitivos de las mismas. 
El análisis estadístico de los datos se ha realizado con el programa estadístico SPSS versión 10.0.7. El estudio de la asociación de las variables categóricas se ha realizado mediante tablas de contingencia y la corrección de Yates al estadístico chi-cuadrado. El análisis del comportamiento de una variable cuantitativa en los grupos dados por una variable categórica se ha realizado mediante la prueba no paramétrica para muestras independientes de Mann-Whitney. Se han considerado significativos p-valores asociados al estadístico de contraste menores que 0,05 , trabajando siempre con un nivel de confianza del $95 \%$.

\section{RESULTADOS}

Durante el periodo de estudio la temperatura media máxima fue de $26,1{ }^{\circ} \mathrm{C}$ (rango $15,8-35{ }^{\circ} \mathrm{C}$ ), media de $4,1{ }^{\circ} \mathrm{C}$ por encima de la habitual, con una humedad relativa del $84 \%$, media de $3 \%$ por debajo de la habitual. Las curvas de temperatura en el periodo a estudio y los años anteriores se expresan en la figura 1.

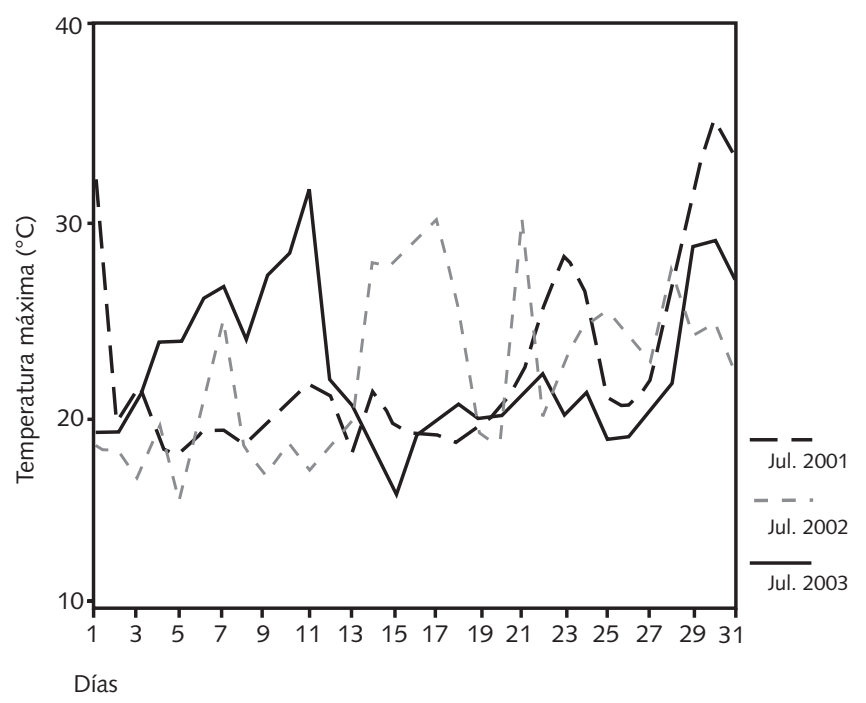

Fig. 1. Evolución de la temperatura máxima del mes de julio durante los años 2001, 2002 y 2003 en Riveira (A Coruña).

En este periodo el número total de pacientes hospitalizados fue de 137. No hubo reingresos en este plazo. De ellos, 87 $(63,5 \%)$ eran varones. El rango de edades fue entre 16 y 93 años, media 71,6 años. Los pacientes en los que se concluyó que el exceso de calor se relacionaba con el motivo de su ingreso fueron $54(38,7 \%)$, de edades comprendidas entre 24 y 93 años (media 74 años), de ellos 38 (70,4\%) varones. Los datos epidemiológicos están recogidos en la tabla II.

Las patologías de base más frecuentes en los pacientes ingresados se recogen en la tabla III.

Los pacientes fallecidos durante este periodo fueron 16 $(11,7 \%)$ [en 1999 fallecieron $4(2 \%)$, en 2000 fallecieron 3 $(3,2 \%)$, en 2001 fallecieron $4(5,1 \%)$ y en 2002 fallecieron 2 $(1,9 \%)$ en este mismo periodo de tiempo]. Esto supone pues un incremento global en la mortalidad de más del 200\%. En la valoración de la mortalidad en cada grupo, en aquellos

\begin{tabular}{|c|c|c|}
\hline \multicolumn{3}{|c|}{ TABLA II } \\
\hline \multicolumn{3}{|c|}{ DATOS EPIDEMIOLÓGICOS. POBLACIÓN A ESTUDIO } \\
\hline & Total & $\begin{array}{l}\text { Patología } \\
\text { por calor }\end{array}$ \\
\hline \multicolumn{3}{|l|}{ Sexo } \\
\hline Varón & 87 & 38 \\
\hline Mujer & 50 & 16 \\
\hline Exitus & 16 & 8 \\
\hline Edad (años) & 71,7 & 74 \\
\hline Estancia media (días) & $7,7(+/-4,76)$ & $8,39(+/-5,5)$ \\
\hline \multicolumn{3}{|l|}{ Patología de base } \\
\hline HTA & $56(41 \%)$ & $20(37 \%)$ \\
\hline Cardiopatía & $53(38 \%)$ & $25(46,3 \%)$ \\
\hline EPOC & $59(43 \%)$ & $33(66,7 \%)$ \\
\hline Nefropatía & $29(14 \%)$ & $11(20,4 \%)$ \\
\hline DM & $30(21 \%)$ & $14(25,9 \%)$ \\
\hline Obesidad & $23(16 \%)$ & $14(25,9 \%)$ \\
\hline Pat. cutánea & $5(3,6 \%)$ & $4(7,4 \%)$ \\
\hline Etilismo & $24(17,5 \%)$ & $9(16,7 \%)$ \\
\hline Neoplasia & $34(24,8 \%)$ & $16(29,6 \%)$ \\
\hline
\end{tabular}

Total: total pacientes ingresados. Patología por calor: pacientes ingresados en relación con el efecto del calor.

\begin{tabular}{|c|c|c|}
\hline \multicolumn{3}{|c|}{ TABLA III } \\
\hline \multicolumn{3}{|c|}{ RESULTADOS ANALÍTICOS. PACIENTES ESTUDIO } \\
\hline & Total & $\begin{array}{l}\text { Patología } \\
\text { por calor }\end{array}$ \\
\hline Temperatura & $36,9(+/-1,14)$ & $36,6(+/-1,17)$ \\
\hline Glucosa & 174 & 194 \\
\hline Creatinina & 1,8 & 1,48 \\
\hline AST & 101,59 & 127,13 \\
\hline ALT & 113,68 & 122,63 \\
\hline GGT & 136,11 & 120,51 \\
\hline FA & 423 & 379 \\
\hline Urea & 70,85 & 68 \\
\hline Sodio & 139 & 142 \\
\hline Potasio & 5,1 & 4,1 \\
\hline $\mathrm{pH}$ & 7,42 & 7,41 \\
\hline $\mathrm{pCO}_{2}$ & 43 & 44 \\
\hline Hematocrito & 37 & 40,3 \\
\hline Cloro & 90 & 88 \\
\hline
\end{tabular}

Total: total pacientes ingresados. Patología por calor: pacientes ingresados en relación con el efecto del calor.

pacientes ingresados por exceso de calor (54 pacientes) la mortalidad es del $12,5 \%$ (8 pacientes) frente a una mortalidad del $10,5 \%$ (otros 8 pacientes), del resto de los ingresados (84), pero no se aprecian diferencias estadísticamente significativas (p 0,32).

En cuanto al estudio estadístico de factores de riesgo asociados al calor en la población ingresada, mediante la corrección por continuidad (Tabla III), vemos que se rechazan las hipótesis nulas de independencia únicamente entre dos variables por lo que se concluye que la patología pulmonar y la obesidad influyen en el agravamiento. Utilizamos el 


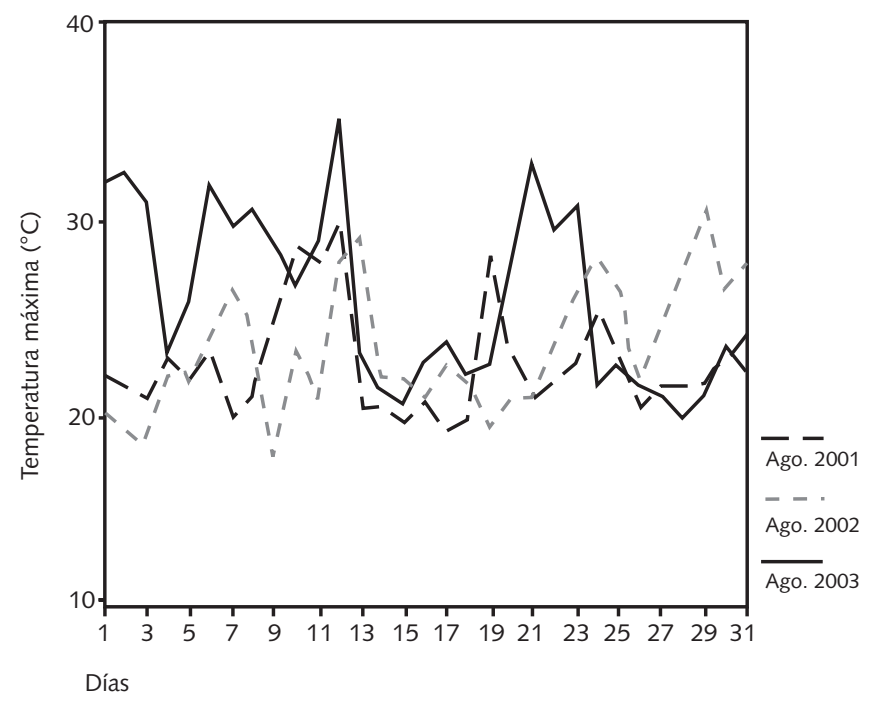

Fig. 2. Evolución de la temperatura máxima del mes de agosto durante los años 2001, 2002 y 2003 en Riveira (A Coruña).

\begin{tabular}{lc}
\multicolumn{2}{c}{ TABLA IV } \\
\begin{tabular}{lc}
\multicolumn{2}{c}{ VARIABLES ANALIZADAS COMO POSIBLES FACTORES DE } \\
RIESGO
\end{tabular} \\
\hline Variable & $p$ \\
\hline Sexo & $>0,05$ \\
Edad & $>0,05$ \\
Patología cardiaca & $>0,05$ \\
HTA & $>0,05$ \\
Patología pulmonar & 0,039 \\
Patología renal & $>0,05$ \\
DM & $>0,05$ \\
Obesidad & 0,017 \\
Patología cutánea & $>0,05$ \\
Etilismo & $>0,05$ \\
Fármacos & $>0,05$ \\
Neoplasia & $>0,05$ \\
Deshidratación & $>0,05$ \\
\hline
\end{tabular}

HTA: hipertensión arterial; DM: diabetes mellitus. Fármacos: se realizó especial seguimiento de aquellos reconocidos como relacionados con empeoramiento (ver tabla I)

coeficiente riesgo relativo para saber en qué consiste la dependencia entre las variables. Para los pacientes con patología pulmonar, la proporción de casos en los que ha habido agravamiento ha sido del 0,61 mientras que en los que no tenían dicha patología ha sido del 0,22. Luego, en los casos en que ha habido agravamiento por calor, la proporción de casos que tenían patología pulmonar es 2,8 veces superior a la proporción de casos que no mostraban la patología. Por otro lado, en los casos en que no ha habido agravamiento, dicha proporción es igual a 0,5. En otras palabras, la patología pulmonar ha provocado un mayor número de agrava- mientos. En cuanto a la variable obesidad, mediante la corrección por continuidad vemos que se rechaza la hipótesis nula de independencia entre variables por lo que se concluye que la obesidad influye en el agravamiento. Utilizando igualmente el coeficiente riesgo relativo para saber en qué consiste la dependencia entre las variables, para los pacientes con obesidad, la proporción de casos en los que ha habido agravamiento ha sido del 0,61 mientras que en los que no eran obesos ha sido del 0,34. Luego, en los casos en que ha habido agravamiento por calor, la proporción de casos que mostraban obesidad es 1,8 veces superior a la proporción de casos que no presentaban obesidad. Por otro lado, en los casos en que no ha habido agravamiento, dicha proporción es igual a 0,6. En otras palabras, la obesidad ha provocado un mayor número de agravamientos.

Comparando las proporciones, también se observa que resulta más influyente en el agravamiento la patología pulmonar que la obesidad.

No se apreciaron diferencias significativas en el resto de los parámetros estudiados. Tampoco resultaron significativas las diferencias entre los datos analíticos de la población total y los pacientes ingresados por empeoramiento relacionado con el calor.

\section{DISCUSIÓN}

La patología relacionada con alteraciones en el ambiente es muy relevante tal y como está documentado en distintos trabajos (9). Los efectos directos del exceso de calor son sin embargo difíciles de evaluar ya que incluso en situaciones clínicas muy concretas y bien definidas como el llamado golpe de calor, el diagnóstico puede pasar inadvertido $(3,4)$. Durante el pasado mes de agosto de 2003 se produjo en Europa una ola de calor con repercusiones en algunos sistemas sanitarios como el francés, estimadas en un exceso de mortalidad de 11.435 casos, afectando en el 69\% de los casos a paciente mayores de 75 años (16). En nuestro país los datos facilitados por en Ministerio de Sanidad indican que las principales causas de muerte durante agosto de 2003 son las mismas que en meses previos estimándose según datos de las Comunidades Autónomas sobre la mortalidad específica durante los meses de julio y agosto, 59 casos de fallecimientos por golpe de calor y 82 por agravamiento de patología previa (17). La práctica clínica y los resultados del estudio que analizamos, parecen indicar posiblemente una mayor incidencia del impacto de la patología relacionada con el calor que la recogida oficialmente, aunque los datos oficiales definitivos están pendientes de publicación. Nuestros datos parece indicar un exceso en la morbimortalidad relacionada con la ola de calor, llegando hasta 54 el número de pacientes ingresados por este fenómeno $(38,7 \%)$ en nuestro centro, y con una mortalidad alta en este grupo (8 pacientes, $12,5 \%)$ frente a 8 pacientes entre los otros $84(10,7 \%)$, aunque no significativa estadísticamente. En cualquier caso, sí existe un notable incremento en la mortalidad global frente a años anteriores $(11,7 \%$ durante la ola de calor frente a 2 , $3,2,5,1$ y $1,9 \%$, respectivamente, durante el mismo periodo de tiempo de los años 1999, 2000, 12001 y 2002). En todos los estudios epidemiológicos realizados se asignan mortalidades atribuidas o excesos de mortalidad extrayendo de la mortalidad observada la esperada. La mortalidad esperada se 
estima en función de una variedad de medidas incluidas las medias de mortalidad en similares periodos de tiempo previos, con métodos diferente en casi todos haciéndose difícil su comparación (18), por lo que no hemos realizado esta valoración aunque el incremento en la mortalidad es evidente. Nuestros datos son difíciles de extrapolar y el estudio tiene algunas limitaciones. Las referidas a la dificultad de la puesta en relación del exceso de calor con algunos ingresos, creemos que se justifica por el incremento notable en los mismos con respecto a años anteriores. No existe evidencia definitiva del papel de este agente físico sobre el claro exceso de mortalidad, pero nos parece una cifra muy significativa como para atribuirlo exclusivamente al azar, especialmente en relación a las cifras previas. Nuestra población, cumple por sus características de edad y patologías de base en muchos casos con los factores de riesgo en lo que respecta al calor. La incidencia de estas patologías es además muy prevalente y sitúa a nuestros pacientes en el perfil de susceptibilidad reconocido al efecto de agentes físicos, lo que hace que esta influencia climática deba ser tenida en cuenta especialmente en los meses de mayor exposición solar. Los estudios epidemiológicos disponibles no atribuyen diferencias en cuanto al riesgo entre hombres y mujeres, nuestro estudio tampoco las aprecia. Varios estudios han reconocido como factor de riesgo la institucionalización de ancianos en residencias (19). También es cierto que en nuestra área sanitaria no existen grandes núcleos de población como los que también están relacionados con aumento en el impacto del calor y especialmente en la mortalidad (18), por lo que éste exceso de mortalidad parece también significativo. Si bien la circunstancia climática que aconteció el pasado verano es según los meteorólogos infrecuente, no es menos cierto que según estos también existe una evidencia de calentamiento atmosférico que posiblemente haga pensar en una mayor incidencia de circunstancias similares en un futuro próximo. Las estrategias culturales de protección frente a temperaturas extremas (ventilación adecuada en los hogares, distribución de horarios con descansos en las horas de máxima intensidad de calor), permiten una adaptación fisiológica a estas situaciones (11). Pero la protección a determinados colectivos debe tratar de garantizarse. La información disponible por la Organización Mundial de la Salud (OMS), indica que en la Región Europea sólo dos ciudades (Lisboa y Roma) cuentan con sistemas de vigilancia y alerta del impacto de exceso de calor. Están en marcha proyectos similares en Barcelona, Budapest, Londres y París (20). La OMS indica que la evidencia parece apuntar a una infravaloración de la importancia y el riesgo sanitario de las olas de calor, con resultados dramáticos como en el caso de la región de París en el verano de 2003, por lo que su conocimiento, evaluación y puesta en marcha de medidas paliativas está fuertemente recomendado por esta organización (18). Así entre otras cabría reseñar: en primer lugar la puesta en marcha de mecanismos de alerta para detección de estos fenómenos climatológicos, medidas de reducción del estrés por calor, ambientes de exposición solar y cerrados (en ambientes cerrados la temperatura óptima debería estar en torno a los $18-24{ }^{\circ} \mathrm{C}$ ), recomendaciones sobre medidas individuales en situaciones de calor extremo (Tabla V) (21). El

\section{TABLA V}

MEDIDAS INDIVIDUALES DE PREVENCIÓN DEL IMPACTO DE CALOR (21)

Recomendaciones generales:

- Aumentar la ingesta de líquidos (no alcohol), independientemente del grado de actividad física. No esperar a tener sed para beber. Si tuviera restricciones hídricas por prescripción facultativa, consultar el incremento de líquidos posible.

-No beber líquidos que contengan cafeína, alcohol o grandes cantidades de azúcar. También evitar bebidas extremadamente frías.

-Permanecer dentro de locales y si es posible con aire acondicionado. Si no se dispone de éste, procurar exposición al menos unas horas al días en ambientes públicos (cines, centros comerciales,...) que dispongan de éste.

- Los ventiladores eléctricos pueden proporcionar algo de confort pero en temperaturas mayores de $35^{\circ} \mathrm{C}$ éstos no previenen enfermedades relacionadas con calor. Tomar duchas 0 baños fríos o buscar ambientes con aire acondicionado es más eficaz.

- Vestir prendas livianas, de colores claros y de fácil retirada.

- Nunca dejar a nadie en vehículos aparcados cerrados.

-Aunque cualquiera puede sufrir enfermedades relacionadas con calor, especialmente en los mayores de 65 años, enfermos mentales, y enfermos crónicos, los cardiópatas e hipertensos, son más susceptibles.

- Visitar a los adultos en situación de riesgo que vivan solos al menos 2 veces al día y vigilar estrechamente signos de enfermedad relacionada con calor. En niños y adultos jóvenes vigilancia aún mas estrecha y continuada.

En caso de exposición obligada al exterior

- Limitar la actividad en el exterior al inicio del día y las horas del final de la tarde.

- Suspender el ejercicio. Si se debe realizar esfuerzos físicos, beber 2-4 vasos de líquidos (no alcohol) fríos cada hora. Bebidas isotónicas pueden reponer las pérdidas de sales y minerales por el sudor. Precaución, si se está bajo restricción hidrosalina consultar con facultativo.

- Procurar descansar a menudo en áreas con sombra.

- Protegerse del sol con sombreros de ala ancha, gafas de sol y aplicar protector solar (al menos factor de protección 15).

pasado 17 de mayo de 2004 el Ministerio de Sanidad y consumo anunciaba un plan de prevención anticipándose a una posible ola de calor, dotándolo con 2,67 millones de euros. Está previsto que se active anualmente entre el 1 de junio y el 1 de octubre. Se crea una Comisión Interministerial (Ministerios de Sanidad, Trabajo y Asuntos Sociales, Interior y Medio Ambiente) y se prevé un nuevo sistema de Información y Vigilancia con distintos niveles de activación de alertas similar a los que están en funcionamiento, mencionados anteriormente, con lo que parece que las tan necesarias medidas para minimizar los riesgos en un futuro próximo, podrían estar comenzándose a tomar (22). 


\section{Bibliografía}

1. Battestini Pons R. Enfermedades por agentes físicos. Introducción. En: Farreras-Rozmán. Medicina Interna. 13ª edición. Barcelona: Ediciones Doyma, 1995, p. 2625.

2. Donoghue ER, et al Criteria for the diagnosis of heat-related deaths. Am J Foren Pathol 1997; 18: 11-14.

3. Bouchama A, Knochel JP. Heat Stroke. N Eng J Med 2002; 346: 1978 1988.

4. Battestini Pons R, Betés de Toro M, Gómez Huelgas R, San Román Terán CM. Enfermedades por agentes físicos. Efectos nocivos causados por la luz y las radiaciones. En: Farreras-Rozmán Medicina Interna. 13a Edición. Barcelona: Ediciones Doyma 1995). p. 2636-49.

5. Keatinge WR, Coleshaw SRK, Easton JC, Cotter F, Mattock MB, Chelliah R. Increased platelet and red cell counts, blood viscosity, and plasma colesterol level during heat stress and mortality from coronary and cerebral trombosis Am J Med 1986; 81: 795-800.

6. Walter JS, Barnes SB. Urgencias por calor de. En: Tintinalli JE. Medicina de Urgencias. 5a Edición. Ed. McGraw-Hill 1999. p. 1405-1413.

7. Lage Ferrón MB, Díaz Jiménez J, Gestal Otero. Influencia de los factores ambientales en el número de ingresos por urgencias en el Complejo Hospitalario Juan Canalejo de A Coruña: elaboración de un modelo de predicción. Rev Esp Salud Pública 1999; 73: 45-60.

8. Hume M Jenkins G, Brooks N, Cresswell D, Doherty R, Durmna C, What is happening to global climate and why? En: Maynard L, ed. Health effects of climate change in the UK. Londres: Department of Health, 2001: 18-47.

9. Heat related Deaths-Chicago, Illinois, 1996-2001 and United States 1979-1999. MMWR (en linea) 2003 july 4 (fecha de acceso 5 diciembre 2003); 52 (26) URL disponible en http://www.cdc.gov/mmwr/preview/mmwrhtml/mm5226a2.htm

10. Luter Barcher J, Dietrich, D Xoplaki E, Grosjean M, Wanner H. European Seasonal and Annual Temperature variability, trends and extremes since 1500. Science 2004; 303: 1499-1503.

11. Keatinge WR, Donaldson GC, Cordioli E, Kunst AE, Mackenbach JP, Nayha S, Vuori J. Heat related mortality in warm and cold regions of Europe: observational study. BMJ 2000; 321: 670-4.

12. The health impacts of 2003 summer heat-waves. Briefing note for the
Delegations of the fifty-third session of the WHO Regional Committee for Europe. World Health Organization Europe 2003.

13. Mackenbach JP, Kunst AE, Looman CWN. Seasonal variation in mortality in The Netherlands. J Epidemiol Commun Health 1992; 46: 261-65.

14. Donalson G, Kovats RS, Keatinge WR, Mc Michael RJ. Heat and cold related mortality and morbidity and climate change. En: Maynard RL. Heat effects of climate change in the UK. Londres. Department of Health United Kingdom; 2001: p. 70-80.

15. Ballester F, Corella D, Pérez-Hollos S, Sáez M, Hervás A. Mortality as a function of temperature. A Study in Valencia, Spain 1991-1993. Intern J Epidemiol 1997; 28: 551-561.

16. Impact Sanitaire de la vague de chaleur en France survenue en août 2003. Rapport d. étape 29 août 2003. Saint-Maurice, Institut de Veille Sanita iré, 2003.

17. Ministerio de Sanidad y Consumo. Informe sobre el potencial impacto sanitario de la ola de calor y la evolución reciente de la mortalidad general por causas en España. Consultado por última vez el 21/05/2004 URL disponible en: URL disponible en: http://www.msc.es/notas_prensa/2003-09-17-2.htm

18. The health impacts of 2003 summer heat-waves. Briefing note for the Delegations of the fifty-third session of the WHO Regional Committee for Europe. World Health Organization Europe.

19. Pajares S, Díaz J, Montero JC, Alberdi JC, Mirón IJ. Mortalidad diaria en la Comunidad de Madrid durante el periodo 1986-1991 para el grupo de 45 años a 65 años: su relación con la temperatura del aire. Rev Esp Salud Publica 1997; 71: 149-160.

20. Extreme weather events: health effects and public health measures. Fact sheet EURO/04/03. Copenhagen, Rome 29 September 2003. World Health Organization Europe.

21. Tips on preventing and managing heat. CDC (en linea)(último acceso 11 diciembre 2003);URL disponible en: http://www.cdc.gov/nceh/hsb/extremeheat/hetattips.htm.

22. El gobierno se anticipa a una posible ola de calor con la puesta en marcha de un plan de prevención. Ministerio de Sanidad y Consumo 17 de mayo; consultado por última vez el 21/05/2004). URL disponible en: http://www.msc.es/notas_prensa/2004-17-05-2.htm. 